\title{
Adenda: Los poliquetos como indicadores biológicos en Latinoamérica y el Caribe
}

\author{
Rodolfo Elías*, Nuria Méndez, Pablo Muniz, Rossana Cabanillas, Carlos GutiérRez-Rojas, \\ Nicolas Rozbaczylo, Mario H. Londoño-Mesa, Paulina Javiera Gárate Contreras, \\ Maritza Cárdenas-Calle, Francisco Villamar, Juan J. A. Laverde-Castillo, Kalina M. Brauko, \\ mariana Araki Braga, Paulo da Cunha lana y Oscar Díaz-Díaz
}
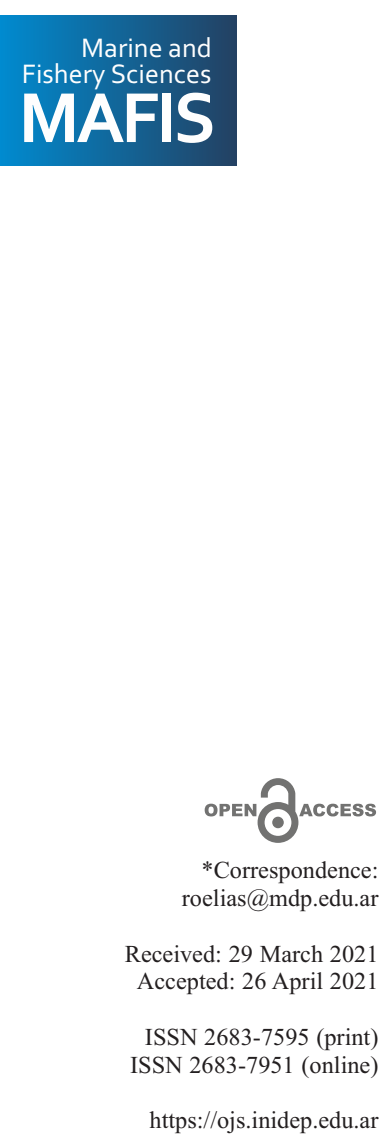

Journal of the Instituto Nacional de Investigación y Desarrollo Pesquero (INIDEP)

This work is licensed under a Creative Commons Attribution-

NonCommercial-ShareAlike 4.0 International License

\begin{abstract}
Adenda a:
Los poliquetos como indicadores biológicos en Latinoamérica y el Caribe por Rodolfo Elías, Nuria Méndez, Pablo Muniz, Rossana Cabanillas, Carlos Gutiérrez-Rojas, Nicolas Rozbaczylo, Mario H. Londoño-Mesa, Paulina Javiera Gárate Contreras, Maritza Cárdenas-Calle, Francisco Villamar, Juan J. Laverde-Castillo, Kalina Brauko, Mariana Araki Braga, Paulo Lana, Oscar Díaz-Díaz (2020). Mar Fish Sci. 34 (1). https://doi.org/10.47193/mafis.3412021010301

Razón de la adenda:

En la elaboración del manuscrito original no se tuvo en cuenta un trabajo científico que trata precisamente de los poliquetos como indicadores ambientales en la Patagonia. Siendo este el primer y único trabajo sobre el tema, era justo y necesario incluir esta información.
\end{abstract}

Addendum to:

Polychaetes as biological indicators in Latin America and the Caribbean by Rodolfo Elías, Nuria Méndez, Pablo Muniz, Rossana Cabanillas, Carlos Gutiérrez-Rojas, Nicolas Rozbaczylo, Mario H. Londoño-Mesa, Paulina Javiera Gárate Contreras, Maritza Cárdenas-Calle, Francisco Villamar, Juan J. Laverde-Castillo, Kalina Brauko, Mariana Araki Braga, Paulo Lana, Oscar Díaz-Díaz (2020). Mar Fish Sci. 34 (1). https://doi.org/10.47193/mafis.3412021010301

Reason for addendum:

During the preparation of the original manuscript, a scientific work that dealt precisely with Polychaeta as environmental indicators in Patagonia was not considered. This being the first and only work regarding this subject, it was reasonable and necessary to incorporate this information.

En sedimentos provenientes de Caleta Valdés $\left(42^{\circ} 15^{\prime} 53^{\prime \prime} \mathrm{S}, 63^{\circ} 40^{\prime} 50^{\prime \prime}\right.$ W) (Península Valdés, Patagonia argentina), se evaluaron los efectos de dos concentraciones de petróleo crudo (tipo Escalante) $\left(1 \mathrm{~g} \mathrm{~kg}^{-1}\right.$ y $\left.20 \mathrm{~g} \mathrm{~kg}^{-1}\right)$ sobre la estructura comunitaria y la actividad de bioturbación (Ferrando et al. 2015). Los sedimentos fueron contaminados en el laboratorio e incubados durante 30 días. Se registró una progresiva disminución en la densidad y la riqueza específica en ambas concentraciones, sin embargo el efecto fue mayor (40 y 55\%, respectivamente) en los sedimentos más contaminados. Asimismo, los coefi- 
cientes de biodifusión $\left(D_{b}\right)$ y bioadvección $(r)$ calculados para la experiencia completa y para los últimos 15 días, fueron casi nulos en estas condiciones experimentales. Axiothella sp. (Maldanidae) mostró cierta sensibilidad al agregado de petróleo, mientras que Cerathocephale sp. (Nereididade) fue tolerante. Resultados similares fueron encontrados en sedimentos provenientes de Caleta Malaspina (45 $\left.8.616^{\prime} \mathrm{S}-66^{\circ} 34.793^{\prime} \mathrm{W}\right)$ y

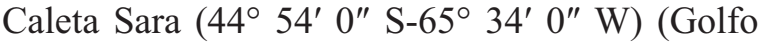
San Jorge, Patagonia argentina) aplicando una metodología similar a la del estudio anterior. En Caleta Malaspina, Glyceridae fue la familia más sensible, mientras que Spionidae, Capitellidae, Maldanidae, Hesionidae, Paraonidae, Syllidae, Orbiniidae, Nereididae, Sabellidae, Dorvilleidae, Onuphidae y Cirratulidae fueron tolerantes a la adición de este contaminante. Dentro de este grupo, Spionidae, Capitellidae y Maldanidae fueron las más abundantes en los sedimentos más contaminados (Ferrando 2015). De manera similar, Capitella sp. (Capitellidae) y Boccardia sp. (Spionidae) predominaron en Caleta Sara en dichas condiciones (Romanut 2019). Además, se evaluó la respuesta a nivel específico de los espiónidos registrados en Caleta Malaspina y se encontraron especies sensibles como Scolelepis (Parascolelepis) sp. y Rhynchospio glutaea mientras que Boccardia proboscidea mostró una alta resistencia a la presencia de petróleo crudo en los sedimentos (Ferrando et al. 2019).

\section{REFERENCIAS}

FERRANDO A. 2015. Biodiversidad de la macrofauna bentónica marina en la zona norte del golfo San Jorge (Patagonia, Argentina) [tesis de doctoral]. Mar del Plata: Facultad de Ciencias Exactas y Naturales, Universidad Nacional de Mar del Plata. 149 p.

Romanut O. 2019. Respuesta de los ensambles de poliquetos y crustáceos de fondo blando ante el agregado de petróleo crudo a los sedimentos (Caleta Sara, Patagonia Argentina) [tesis de licenciatura]. Universidad Nacional de la Patagonia San Juan Bosco. 47 p. 